\title{
La SGIM-Foundation: un Update et un appel à projets
}

\section{Jean-Michel Gaspoz}

Prof. Dr, Président du Directoire de la Société Suisse de Médecine Interne Générale SSMI
Correspondance: SGIM-Foundation Solothurnerstrasse 68 Case postale 422 CH-4008 Bâle
Le 12 avril 2010, les Professeurs Ulrich Bürgi et Verena Briner, de même que les Docteurs Werner Bauer, Jürg Pfisterer, Romeo Providoli, Susanna Stöhr et Regula Sievers, ont légalement constitué, à Berne, la SGIM-Foundation, une Fondation de la Société Suisse de Médecine Interne Générale (SSMI). Le capital initial de la Fondation, de 50000 francs, a été complété par des dons de l'association des internistes de Zurich (VZI) et de la Société Suisse de Médecine Interne Générale. Des Sociétés cantonales de médecine, des sponsors de l'industrie et des sponsors privés sont sollicités pour régulièrement alimenter ce fonds et compléter ce que la SSMI elle-même est à même d'y déposer.

\section{But de la Fondation}

Le But de la Fondation est le développement de la recherche et de l'enseignement dans le domaine de la médecine interne générale. C'est-à-dire:

- Soutien à des projets de recherches; ces projets peuvent relever de la recherche clinique, de l'épidémiologie clinique, ou de la recherche sur les services de santé et l'organisation des soins.

- Remise de prix et de bourses;

- Soutien à la mise sur pied ou à la conduite de projets dans le domaine de formations pré-graduées, post-graduées et continues;

- Soutien à la mise sur pied d'interventions en faveur des patients.

D'autres domaines peuvent être couverts par la Fondation, ainsi que d'autres buts.

\section{Organes de la Fondation}

Les organes de la Fondation sont constitués par son Conseil de fondation, le Secrétaire Général de la Société et l'Organe de révision. Le Conseil de Fondation est formé de cinq à sept membres; le Président de la société, ainsi qu'au minimum un membre du Conseil de Fondation de la société, doivent en être membre. Le Conseil de Fondation est compétent pour accueillir de nouveaux membres ou remplacer ses membres.

\section{Le Conseil de Fondation en 2012}

Suite au départ du Prof. Ulrich Burgi et de la Dre Regula Sievers, le Conseil de Fondation est constitué du Professeur Jean-Michel Gaspoz, Président, du Dr Jürg Pfisterer, Vice-Président, et des Docteur Werner Bauer, Professeur Verena Briner, Docteur Romeo Providoli, Docteur Susanna Stöhr et de Monsieur Lukas Zemp, secrétaire général de la Société.

\section{Appel d'offres \\ Chaque année, la Société Suisse de Médecine Interne Générale procédera à un appel d'offre de projets ou de bourses concernant un thème donné. Ces thèmes seront en lien direct avec les problématiques scienti- fiques actuelles en médecine interne générale et avec l'organisation des soins dans cette discipline. Les projets seront examinés par un Comité scientifique réunissant des autorités médicales et universitaires de Suisse.}

\section{Appel à projets 2012/2013 pour la SGIM-Foundation}

\section{Organisation des soins: transition entre l'hôpital et la communauté}

La SGIM-Foundation lance un appel à des projets étudiant les problèmes de transition et de discontinuité des soins au moment de la sortie de l'hôpital et proposant des solutions innovantes pour les résoudre. Seront privilégiés les projets proposant ou testant des solutions qui pourraient être promues par la Société Suisse de Médecine Interne Générale et mis en pratique dans l'ensemble de la Suisse. Ainsi, une aide financière pourra être sollicitée aussi bien pour la préparation que pour la réalisation de tels projets. Les projets devront être construits selon une méthodologie rigoureuse permettant, à terme, l'évaluation de leur impact.

Maximum trois projets seront financés, à hauteur de 70000 francs chacun. Ils seront évalués par un Comité scientifique, formés de 4 experts dans le domaine.

Les projets devront être envoyés au plus tard le 31 janvier 2013 à la SGIM-Foundation, Solothurnerstrasse 68, Case postale 422, 4008 Bâle. 


\section{Thème 2012/2013}

Lors du symposium Platine 2011 à Thun, les participants se sont penchés sur les écarts et les fissures dont souffre notre médecine moderne. Un important débat a concerné les fossés qui séparent l'hôpital et la ville, tout particulièrement au moment de la sortie d'un patient de l'hôpital. Cette dernière faille a été intitulée «Lost in transition» par Dr Eric A. Coleman, hôpitaux selon le système des DRGs, qui aura pour conséquence un raccourcissement des durées de séjour hospitaliers et le retour à domicile de patients plus instables; la nouvelle ordonnance fédérale sur les «Soins Aigus de Transition», qui confie aux médecins hospitaliers la prescription des soins infirmiers des deux premières semaines suivant la sortie de l'hôpital.

\section{Ces thèmes seront en lien direct avec les problématiques scientifiques actuelles en médecine interne générale et avec l'organisation des soins dans cette discipline.}

Associate Professor of Medicine, University of Colorado, Health Sciences Center. Combien d'informations sont non communiquées ou perdues entre les médecins hospitaliers et les praticiens, ou l'inverse? Comme conséquence, 19\% des patients sortis d'un hôpital sont victimes d'un incident dans les 3 semaines suivantes, dont $66 \%$ liés aux médicaments; en outre, $1 / 3$ des patients hospitalisés pour insuffisance cardiaque sont réadmis dans les 30 jours. Deux éléments rendent la résolution de cette problématique d'une urgente actualité: le remboursement des
Aussi, la Société Suisse de Médecine Interne Générale a-t-elle décidé de faire de la problématique «Lost in transition» son thème 2012/2013. En effet, la Société contient en son sein les principaux acteurs de cette problématique, c'est-à-dire les médecins hospitaliers et les praticiens, et se trouve particulièrement bien placée pour lui trouver des solutions.

Vous trouverez de plus amples informations sous: www.sgim.ch/fr/prixsoutien-a-la-recherche/ sgim-foundation

\section{Vous qui lisez une revue des Editions médicales suisses,}

\section{saviez-vous que ...}

- les Editions médicales suisses sont une coopération entre la Fédération des médecins suisses (FMH) et la plus ancienne maison d'édition et imprimerie au monde (les Editions Schwabe, fondées en 1488)?

n que les EMH sont les éditions de pointe en Suisse dans le domaine des journaux médicaux, avec dix revues spécialisées, des articles paraissant intégralement en ligne et un choix de livres de plus en plus large?

- que toutes les revues paraissant aux EMH sont les organes officiels de publication des sociétés médicales correspondantes?

Aux EMH, les articles sont publiés par des médecins pour des médecins. Des comités de rédaction indépendants constitués de spécialistes réputés assurent la qualité et l'actualité des articles qui couvrent toutes les disciplines médicales. Le choix des thèmes reflète les intérêts divers des médecins et des sociétés médicales et ne se concentre pas sur les aspects commerciaux.

Si vous souhaitez en savoir plus sur les EMH, vous trouverez plus d'informations sous www.emh.ch. Vous y trouverez également les revues publiées en ligne et des outils pratiques pour vos recherches d'articles. Nous sommes à votre disposition pour répondre personnellement à vos questions:

EMH Editions médicales suisses SA

Farnsburgerstrasse 8

CH-4132 Muttenz
Marketing et communication

Tél. +41 (0)614678506 / Fax +41 (0)614678556 marketing@emh.ch

EMH Editions médicales suisses - des publications à la pointe de la médecine 